licher Verfahrensaxiome, diese Macht sich im Verfahren nur als Abfolge neutralisierter und entdramatisierter Zugriffe auf das Individuum niederschlagen, Zugriffe, die durch generalisierte Tatbestandsvoraussetzungen gebremst und durch die bedingungslose Beachtung des individuellen Subjekts ihren elementaren organisatorischen Zusammenhang finden. Die zulässige Folgenorientierung der Strafjustiz hat nicht den Inhalt, verdächtige Täter mit denselben Kampfmitteln zu verfolgen, die diese möglicherweise anwenden bzw. die ihnen von den Strafjustizbehörden unterstellt werden. ${ }^{23}$ Die Art und Weise, in der Peter Egloff ohne Chance einer abwehrenden Reaktion »in die Falle geriet, zeigt exemplarisch, wie instrumentelle Justizpolitik ihre eigenen Axiome untergräbt. Treten die Institutionen der Justiz dem Verfahrensunterworfenen auf derselben Ebene der Zweckverfolgung entgegen, auf der sich der Unterworfene legitimerweise bewegt, dann ist leicht abzusehen, wer in diesem ungleichen Kampf die Oberhand behält.

Andreas Zielcke

\title{
Produktionsverhältnisse und staatliches Strafen. Zur aktuellen Diskussion über Rusche/ Kirchheimer*
}

Nachdem die Übersetzung des Buches von Rusche und Kirchheimer ( $\mathrm{R}$ \& $\mathrm{K}$ ) "Sozialstruktur und Strafvollzug * (engl.: Punishment and Social Structure, 1939) vor sechs Jahren einhellig begrüßt worden war, und auch in dieser Zeitschrift als »unerläßliche Voraussetzung für die Diskussion einer politischen Okonomie des Strafrechts (Rausch 1975, S. 218) gefeiert wurde, ist aus R \& K ein Mythos geworden. Sie stehen dafür, daß Gefängnissysteme und Kapitalismus als aufs engste miteinander verwoben anzusehen sind. Sich auf ihren Ansatz zu berufen, macht klar, daß man jedenfalls nicht das Geschäft der Strafvollzugsreformer betreiben will. Insofern wurde das Namenspaar zur lachenden Sonne der Anti-Kerkerbewegung. Damit einher ging leider ein Verzicht auf sorgfältige Lektüre des Buches. Schon gar nicht hat eine Diskussion über die Frage, ob $\mathrm{R} \& \mathrm{~K}$ zur Anleitung weiterer historischer und vergleichender Forschungen eine überzeugende Perspektive anbieten, stattgefunden, mit Ausnahme der erfreulich respektlosen und teilweise treffenden kritischen Beiträge von Steinert und Treiber. Außerhalb des deutschen Sprachraums sind in etwas größerem Umfang, so in England, Italien, Skandinavien und den USA, Diskussionen geführt und veröffentlicht worden; zusammengenommen mögen sie eine differenziertere Einschätzung der Einsichten von R \& K erlauben. Im folgenden versuche ich, diese Gedanken systematisiert darzustellen und eine Zwischenbilanz zu ziehen.

\section{Die Theorie von $R$ \& $K$}

I. Die ökonomische Erklärung von Strafvollzug

$\mathrm{R} \& \mathrm{~K}$ 's Leistung liegt in der ökonomischen Erklärung des gesellschaftlichen Strafsystems, insbesondere der Gestaltung des Strafvollzuges. Erstmals haben sie die

23 Vgl. G. Arzt, a. a. O. (Anm. 18), S. $78 \mathrm{ff}$.

*Für kritische Hinweise zu einer ersten Fassung danke ich Wolfgang Stangl und Michael Voß. 
Diese Arbeit muß heute in zweierlei Hinsicht als Dokument gelten: einerseits birgt sie wichtiges Material zur Frühgeschichte der CDU/CSU, andererseits ist sie ein wissenschaftsgeschichtliches Dokument für die Frühphase der Entwicklung der Wissenschaft von der Politik im Nachkriegsdeutschland.

Aus der Nachscbrift (1954) von A. R. L. Gurland: »Politische Wissenschaft ist keine Zauberei und kein Agglomerat spekulativer Deutungen. Wo ihr das grundlegende historische und soziologische Material abgeht, nützt ihr das Besondere ihrerSicht nicht viel. Sie kann dann Fragen stellen, vermag sie aber nicht zu beantworten. Freilich ist schon die Fragestellung ein nicht unwesentlicher Forschungsbeitrag. Mit Hilfe der Fragen, die die politische Wissenschaft an das Parteigeschehen heranbringt, kann das einer historischen oder soziologischen Bearbeitung noch nicht zugängliche fragmentarische Rohmaterial, das in Hülle und Fülle gehoben werden kann, doch wenigstens als Ma-
A.R.L. Gurland

Die

su

e und Entwicklung

bis 1953

rausgegeben von Dieter Ëring

II HiRt

fip :

Evapestech

terial zur Zeitgeschichte gesiebt, geordnet und gruppiert werden. Es wird in der Regel keine durchgängigen Zusammenhänge erhellen, keine ausgebildeten Strukturen des sozialen Geschehens sichtbar werden lassen; es wird aber viele Episoden in abgerundeter Gestalt herausschälen, zahlreiche Aspekte des Geschehens an vielerlei Schnittstellen aufblitzen lassen.

Man kann solche (. . .) Beiträge (. . .) zu den Acta Historica legen, bis sie alt genug geworden sind, als Geschichtsquellen auch bei den Zünftigen Fußnotengängigkeit $z u$ erlangen. Man kann sie aber auch zu einzelnen Durchblicksbildern zusammenstellen; man kann darüber hinaus den Versuch machen, aus ihnen ein Panorama des um das Untersuchungsobjekt kreisenden Geschehens zu entwerfen und das Panorama vieldimensional zu gestalten, indem man Zeitlängsschnitte, Raumquerschnitte und Situationsreliefbilder aufeinanderprojiziert. Das ist es, was in der vorliegenden CDU-Studie versucht worden ist." 
steten Änderungen im Strafvollzug nicht mit allgemeinen Tendenzen zur Humanisierung oder mit dem Wirken großer Männer (etwa Howard, Wiechern, Krohne usw.) erklärt, sondern mit ökonomischen Strukturen. Daß sie ihre Analyse zudem über ein ganzes Jahrtausend hinweg durchführten, verlieh ihr fast den Schein absoluter Gültigkeit.

Um die inzwischen formulierte Kritik verständlich zu machen, ist es notwendig, die Grundelemente der Theorie von $\mathrm{R} \& \mathrm{~K}$ in Erinnerung zu rufen. Dabei sollen die Gedanken Rusches von denen Kirchheimers abgehoben werden. Kirchheimer hat ja, aufgrund der Notizen Rusches, die in Kapitel 2 bis 8 eingearbeitet wurden, die Endfassung des Buches in New York City allein geschrieben, wobei die Einführung und die Kapitel 9 bis 13 aus seiner Feder stammen. Die Entwürfe Rusches stimmen thematisch wohl weitgehend mit dessen Aufsatz "Arbeitsmarkt und Strafvollzug" (1933) überein. Im Buch sind in die Argumentationskette des Aufsatzes vor allem umfassende historische Belege eingefügt worden. Allerdings scheint Rusche mit der endgültigen Fassung, die seine Gedanken von Kirchheimer erhielten, nicht sehr glücklich gewesen zu sein, wie ein Brief im Pollock-Archiv zeigt (Melossi 1978, S. 83 ).

Die ökonomische Erklärung nennt drei Zusammenhänge, die den Strafvollzug in seiner Gestalt bestimmen:

a) »Alle Bemühungen um die Reform der Behandlung der Verbrecher finden ihre Grenze an der untersten sozial bedeutsamen proletarischen Schicht, die die Gesellschaft von kriminellen Handlungen abhalten will ... (d)enn eine wahrhafte Besserung der Lage der Verbrecher über diese Grenze hinaus würde so weite Schichten nicht mehr vom Verbrechen zurückhalten, daß dadurch jeder mögliche Rahmen eines Strafvollzuges gesprengt wäre* (Rusche 1933, S. 67).

b) Zwischen der Lage auf dem Arbeitsmarkt, die zwischen Arbeiterknappheit und Menschenüberfluß schwanken kann, und der Gestaltung von Strafen und Strafvollzug besteht folgender Zusammenhang: $\gg$ Arbeitslose Massen, die vor Hunger und Not zu Verzweiflungsdelikten neigen, wird man davon nur durch grausame Strafen abhalten können. Am praktikabelsten erscheint in solchen Fällen schwere körperliche Züchtigung der Verbrecher, wenn nicht ihre rücksichtslose Vernichtung. . . . In einer Gesellschaft, in der die Arbeiter knapp sind, wird der Strafvollzug ganz andere Funktionen haben ... Daher besteht in allen Gesellschaften, in denen Arbeitermangel herrscht, eine Tendenz zur Abkehr von Körperstrafen und von der Vernichtung der Verbrecher. Auch der Verbrecher ist als Arbeitskraft noch wertvoll, man tötet ihn nicht gerne, sondern verwertet ihn, wenn es geht. Zwangsarbeit ist das geeignete Strafmittel « (Rusche 1933, S. 68 f.).

c) "Insoweit die Gefangenen nicht dazu benutzt werden, die Lücken auf dem Arbeitsmarkt aufzufüllen, wird die Wahl der Methoden weitgehend durch fiskalische Interessen bestimmt

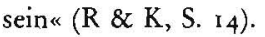

Hervorhebenswert ist, daß These 1, die Verschlechterungsthese, etwa gleichlautend in Buch und Aufsatz formuliert ist (R \& K, S. I3); hingegen fehlt in dem Buch eine knappe Darstellung des Zusammenhangs von Arbeitsmarkt und Strafvollzug (These 2, Arbeitsmarktthese), wennschon man die Kapitel II bis IV als ihre breit angelegte historische Ausarbeitung ansehen kann. Die Fiskalthese (These 3) schließlich fehlt in Rusches Aufsatz gänzlich; sie dürfte allein von Kirchheimer stammen.

2. Die Erklärungskraft der Arbeitsmarktthese (Rusche 1933)

Im Aufsatz von 1933 versucht Rusche die Verschlechterungsthese, die er als eine Aussage "rein formaler Natur " (Rusche 1933, S. 67) wertet, inhaltlich durch die Arbeitsmarktthese auszufüllen. Als einschlägigen Beleg nennt er dabei die Zeit zu Beginn des 17. Jh. - die Epoche der Zuchthausgründungen: die Institutionen sind vor allem Produktionsstätten. Gleichermaßen bedeutsam ist dann die industrielle Revolution, in der massenhaft Arbeiter durch die Maschine freigesetzt werden; hier 
wäre die Rückkehr zu den "mittelalterlichen Methoden « geboten gewesen, aber - und

hier schränkt Rusche die ökonomische Erklärung teilweise ein -:

mes kam nicht so weit, sei es, daß die mühsam erworbenen Ideale der Humanität es verhinderten, sei es, daß politische Klugheit die Herrschenden davor zurückschrecken ließ, durch eine so offene Provokation die ohnehin revolutionäre Situation zu überspannen. Die Freiheitsstrafe blieb erhalten, ein Uberrest aus einer Epoche ganz anderer sozialer Konstellation, nicht zu verstehen aus der Interessenlage der Gegenwart, aber sie wandelte ihre Funktion... Aus sinnvollen Zwangsarbeitsanstalten wurden Zuchthäuser zu Orten bloßer Quälerei« (Rusche 1933, S. 74).

Rusche nennt einen zweiten Beleg für die Arbeitsmarktthese: In den USA unterlag im I9. Jh. das von J. Howard in England ersonnene Einzelhaftsystem, das die Quäker in Philadelphia erstmals verwirklichten, dem Auburn-System, das dem chronischen Arbeitskräftemangel des prosperierenden Kapitalismus Rechnung trug und »die Gefängnisse zu gut rentierenden Produktionsstätten « (Rusche I933, S. 75) machte. Mit Blick auf die Weltwirtschaftskrise der zwanziger Jahre ist Rusche aber wieder bereit, die Geltung seiner These einzuschränken: Die Einführung der Arbeitslosenfürsorge in Deutschland habe diejenigen, die aus dem Produktionsprozeß ausfielen, davor bewahrt, aus Mangel Verbrecher zu werden. Freigestellt von der Aufgabe, die Ärmsten noch abschrecken zu müssen, »brauchten die Reformen des Strafvollzuges ... zunächst nicht aufgegeben zu werden « (Rusche 1933, S. 76). In den USA hingegen brach der »humane» Strafvollzug völlig zusammen, zumal auch die Gewerkschaften Gefangenenarbeit für freie Unternehmer scharf bekämpften und etwa um 1930 vollständig beseitigt hatten (Mitford I97I, S. 214).

\section{Differenzierung der Theorie ( $\mathrm{R} \& \mathrm{~K}$ 1939)}

Die begrenzte Erklärungskraft der Arbeitsmarktthese (triftig für die Entstehung von Zuchthäusern und für den Sieg des Auburn-Systems in den USA, nur eingeschränkt zutreffend für den Strafvollzug im I 8. und 19. Jh. in Europa) mag Kirchheimer bei seiner Uberarbeitung des Buches bewogen haben, dem Verschlechterungsprinzip und dem fiskalischen Argument doch recht großen Wert beizumessen und damit den Ansatz Rusches zugunsten einer differenzierten Deutung zu erweitern.

Das Buch enthält - ähnlich dem Aufsatz Rusches - eine Beschreibung des späten Mittelalters, in dem vom Strafsystem der Geldbuße zum System von Leibes- und Körperstrafen übergegangen wurde, um die Heere besitzloser, im Prozeß der ursprünglichen Akkumulation freigesetzter Menschen zu disziplinieren. Dieser Arbeitskraftüberfluß schlägt erst im Merkantilismus, bei starkem Anwachsen der Produktionsstätten, in einen Arbeitskraftmangel um; infolgedessen entwickeln sich Zuchthäuser, Galeerensklaverei, Deportation in Kolonien (Kapitel 2-4). Kapitel s stellt die Straftheorien in der Zeit der französischen Revolution dar: gegen absolutistische Willkür setzt das erstarkende Bürgertum eigene Philosophien der Gerechtigkeit, vor allem die von Beccaria. In Kapitel 6 wird der Verfall der Haftstrafe zur Körperstrafe durch harte Disziplinierung der Gefangenen, Einführung der Tretmühle, kargste Verpflegung usw. beschrieben. Dies ist Beleg für die Verschlechterungsthese, aber nicht für die Arbeitsmarktthese, die Hinrichtungen erwarten ließ. R \& K schreiben: „Die Haftstrafe wurde genau in dem Augenblick, in welchem die ökonomische Grundlage des Zuchthauses zerstört worden war, zur hauptsächlichen Bestrafungsart der ganzen westlichen Welt« (R \& K, S. 143).

Kapitel 8 nennt mit dem Einzelhaftsystem, soweit es in den USA angewendet und zugunsten des produktionsintensiven Auburn-Systems aufgegeben wurde, den neben der Epoche des Merkantilismus - stärksten Beweis für die Arbeitsmarktthese: In den USA gab es offenbar »einen größeren Bedarf an Arbeitern als es zu irgendeiner 
Zeit während der merkantilistischen Epoche in Europa gegeben hatte ( $R$ \& $K, S$. I 78 f.). Danach ist aber die ökonomische Erklärung allein nicht mehr ausreichend. Im Europa des ausgehenden 19. Jh., bei optimaler Ausnutzung der Arbeitskräfte, wird das Konzept des Stufenstrafvollzuges entwickelt, bei dem gute Arbeit im Gefängnis zum Anlaß für frühere Entlassung wird. Aber dies mag vor allem Disziplinierungstechnik sein (R \& K, S. 217) oder aus fiskalischen Gründen geschehen ( $\&$ \& K, S. 2 I 2). Daß in Deutschland nach dem r. Weltkrieg die Humanisierung des Strafvollzuges während der Weimarer Republik weiterging (Kapitel 9), läßt sich mit dem Zusammenbruch des Arbeitsmarkts in der Weltwirtschaftskrise kaum in Einklang bringen. Als Erklärung hierfür wird das Fiskalargument (R \& K, S. 230) angeboten, das auch die wachsende Prominenz der Geldstrafe im 20. Jh. (Kapitel ıo) erklären soll (R \& K, S. 235, S. 242). Vollends gegenläufig wird in der Zeit des Nationalsozialismus die Entwicklung: wachsendem Arbeitskraftbedarf steht eine Verschärfung des Strafvollzuges gegenüber. Erklärt wird dies mit dem Verschlechterungsprinzip (R \& K, S. 254, S. 263), denn die Lebensverhältnisse der ärmsten arbeitenden Bevölkerung sollen auf einem Minimalniveau gehalten werden. Im Schlußkapitel wird schließlich gezeigt, daß die verschärfte Strafzumessungspolitik des Nationalsozialismus bloß Menschenmißachtung darstellt, nicht aber das rationale Argument für sich in Anspruch nehmen kann, damit Kriminalität abschrecken zu können.

Aus der Durchsicht der Anwendungssituationen für die 3 Thesen von R \& $\mathrm{K}$ ergibt sich als Bilanz (wie die folgende Ubersicht zeigt): Die Arbeitsmarktthese hat vier erfolgreiche Anwendungssituationen, scheitert aber vor allem im Deutschland des 20. Jh. Die Verschlechterungsthese gilt vor allem für die Vollzugsgestaltung nach der industriellen Revolution und im Nationalsozialismus. Die Fiskalthese erlangt Erklärungskraft vor allem in der Weimarer Republik, aber wohl auch schon im Kaiserreich.

Ubersicht:

Okonomische Situation und Strafvollzug in verschiedenen Epochen und Regionen (nach Rusche \& Kirchheimer)

\begin{tabular}{|c|c|c|c|c|c|c|}
\hline \multirow[t]{2}{*}{ Epoche/Region } & \multirow[t]{2}{*}{$\begin{array}{l}\text { R \& K- } \\
\text { Kapitel }\end{array}$} & \multirow[t]{2}{*}{$\begin{array}{l}\text { Arbeits- } \\
\text { kräfte- } \\
\text { mangel? }\end{array}$} & \multirow[t]{2}{*}{ Strafsystem } & \multicolumn{3}{|c|}{$\begin{array}{c}\text { ein- } \\
\text { schlägig } \\
\text { ist These }\end{array}$} \\
\hline & & & & I & & 3 \\
\hline ausgehendes Mittelalter & 2 & nein & Leibes- und Todesstrafe & + & + & - \\
\hline Merkantilismus & 3,4 & ja & $\begin{array}{l}\text { Zucht-, Arb.-häuser, Galee- } \\
\text { re, Deportation }\end{array}$ & - & + & - \\
\hline (Aufklärung) & 5 & $?$ & $\begin{array}{l}\text { Popularisierung v. Beccarias } \\
\text { Straftheorie }\end{array}$ & - & - & - \\
\hline Industrielle Revolution & 6,7 & nein & Haft, Peitsche, Tretmühle & + & - & - \\
\hline USA im 19. Jh. & 8 & ja & $\begin{array}{l}\text { Einzelhaft m. gemeins. Arb. } \\
\text { (Auburnsystem) }\end{array}$ & - & + & - \\
\hline $\begin{array}{l}\text { Europa, Deutschland im } \\
\text { 19. und Beginn 20. Jh. }\end{array}$ & 9,8 & ja & Stufenvollzug & - & + & + \\
\hline Weimarer Rep. & 9,10 & nein & Reformismus, Geldstrafe & - & - & + \\
\hline NS-Zeit & II & $\begin{array}{l}\text { zunehmend } \\
\text { ja }\end{array}$ & $\begin{array}{l}\text { zunehmende Verschärfg. d. } \\
\text { Haftbedg. }\end{array}$ & + & - & - \\
\hline
\end{tabular}

+ einschlägig, - nicht einschlägig

Das Wechseln zwischen den Erklärungen macht die Beziehung der Thesen untereinander deutlich. These 2 erklärt die Strafgestaltung in der Situation mangelnder Arbeitskräfte: mit dem Wert der Arbeitskraft steigt das Niveau, in dem der bestrafte Mensch nicht mißachtet wird. These I erklärt die Strafgestaltung in Situationen des 
Arbeiterüberflusses; die rücksichtslose Senkung der Lebensverhältnisse soll die Armutskriminalität eindämmen. Mit dem Erstarken des zentralen Staates wird der Zusammenhang zwischen Arbeitsmarkt und Strafgestaltung gebrochen durch fiskalische und ideologische Interessen des Staates. So könnte eine erste Würdigung des Buches von $\mathrm{R} \& \mathrm{~K}$ aussehen, bei der die 3 Thesen allerdings nebeneinander stehen und nicht theoretisch verbunden sind.

\section{Kritik der Analyse von $R$ \& $K$}

Kritik an R \& $\mathrm{K}$ wurde in dreierlei Hinsicht geäußert. Erstens gibt es Vorbehalte gegenüber der historischen Korrektheit der Analysen. Bemängelt wird zweitens ein "Okonomismus" in der Analyse, der die Strafgestaltung in bestimmten Epochen nicht richtig erklärlich mache. Und drittens wird das Unvermögen, die Strafentwiccklungen im 20. Jahrhundert zu erklären, betont. Alle drei Ebenen der Kritik unterstreichen die Notwendigkeit erheblicher Verfeinerungen der Theorie. Die umfassendste Würdigung hat dabei Dario Melossi in seiner Einleitung der italienischen Ausgabe des Buches von R \& K unternommen (1978).

I. Zweifel an historischer Genauigkeit

Kritik an der bistorischen Arbeitsweise der Autoren betrifft das ausgehende Mittelalter und den Beginn des Merkantilismus. Steinert und Treiber (1978) bemängeln, daß Quellenkritik fehle, daß zu pauschal über große Zeiträume hinweg argumentiert werde, und daß die unterschiedlichen Entwicklungen, die verschiedene europäische Länder nahmen, zu wenig Beachtung fänden. Konkret machen sie die Kritik an einer Extremformulierung der Arbeitsmarktthese fest, die sie »Ausrottungsthese " nennen: »das Strafrecht dient der Ausrottung der subsistenzlosen Bevölkerungsanteile, die der Arbeitsmarkt nicht aufnehmen kann « (1978, S. 87). Anhand historischer Quellen weisen sie nach, daß der quantitative Umfang von Hinrichtungen nur unerheblich die Zahl der Arbeits- und Besitzlosen vermindert haben konnte. Deshalb ist nach ihrer Meinung diese »wahrscheinlich stärkste mögliche Formulierung des Arbeitsmarkt-Arguments « (1978, S. 87) nicht empirisch beweisbar. Allerdings stellen R \& K eine These dieser Form auch nicht auf; wo von Ausrottung im Kapitel 2 die Rede ist, handelt es sich um Referatstellen aus Werken von Schmidt und v. Hentig (R \& K, S. 29, S. 3I), also nicht um Ableitung aus der Theorie, sondern um empirische Darlegungen aus anderer Feder. Dennoch läßt sich hieran - wie auch an der später zu diskutierenden Studie von Jancovic - ein wichtiger Hinweis auf die Grenzen der Interpretierbarkeit von R \& K's Thesen gewinnen: die Zusammenhänge zwischen Arbeitsmarkt und Strafvollzug sind nicht quantitativ, sondern qualitativ zu verstehen. Es geht nicht, wie etwa auch Ignatieff (1978, S. I 2) irrtümlich vermutet, um die Absorbtion von freigesetzter Arbeitskraft in Zeiten des Überschusses durch Gefängnisse oder gar Hinrichtungen. Es geht vielmehr um die Art, wie Arbeitskraft bewertet wird, ob sie genutzt wird im Strafvollzug oder ob man sie - auch auf die Gefahr ihres Verkommens - ignoriert. Insofern trifft auch die Kritik von Olaussen (1976, S. 36 f.) nicht den Kern, wenn er nachweist, daß in der Epoche des Merkantilismus die Zeiten des Arbeitskraftmangels keineswegs synchron mit den Entstehungszeiten von Zuchthäusern verliefen, daß sie sogar in Deutschland vor und

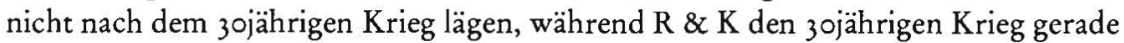
als Illustration für die wachsende Nachfrage nach Arbeitskraft anführen (R \& K, S. 37).

Zusammengenommen deuten die historischen Überprüfungen an, daß der Zusam- 
menhang zwischen Arbeitsmarkt und Strafgestaltung nicht als bloß oder als überwiegend quantitativ bestimmt zu denken ist. Auch Melossi weist auf die qualitative Natur der Kernthese von R \& K hin: Zucht- und Arbeitshäuser konnten seiner Meinung schon ihrer geringen Größe wegen nur einen geringen Beitrag zur besseren Ausnutzung des Arbeitskraftpotentials leisten (1978, S. 80). Sie demonstrierten vielmehr die neue Einschätzung des Menschen als wertvolles Kapital. Man muß also die quantitative Dimension der Arbeitsmarktthese so formulieren: Die Gefängnisse dienen in Zeiten des Arbeitskraftüberflusses nicht der Vernichtung oder Verbergung von freigesetzter Arbeit; wohl aber sollen sie in Zeiten des Arbeitskraftmangels noch freie Arbeitsreserven verwerten.

2. Okonomismus-Vorwurf

Das zweite Argument gegen die Analyse von R \& K, der Okonomismus-Vorwurf, wird in einer anderen Arbeit von Steinert und Treiber zusammen mit Hassemer (1978) formuliert. Mit Blick auf verschiedene historische Epochen räumen die Autoren die Stichhaltigkeit der Theorie lediglich für den Merkantilismus ein, betonen aber ihr Versagen für die Erklärung der Strafphilosophie des Zeitalters der Aufklärung. Wir haben in der Ubersicht diese Epoche, im Kapitel s des Buches beschrieben, in Klammern gesetzt, weil hier nicht von Strafe, sondern von materiellem und formellem Strafrecht die Rede ist, und weil nicht Realien, sondern Ideen zur Debatte stehen. Insofern mag man sich fragen, ob die Kritik der Autoren wirklich gegen R \& K's Theorie, die den Strafvollzug betrifft, vorgebracht werden kann (vgl. auch Melossi 1978, S. 76).

Ferner schließen die drei Autoren aus der unterschiedlichen Karriere, die das Modell der Einzelhaft in Europa und den USA nahm, auf eine wachsende Disparatheit von Wirtschaft und Strafvollzug. Daß überhaupt die im I 8. Jh. in England entwickelte Einzelhaft (also Totaleinschließung) in die USA eingeführt wurde, deute »wirtschaftliche und fiskalische Losgelöstheit des Strafvollzuges " (Hassemer, Steinert, Treiber 1978, S. 38) an. Der rasche Niedergang der Einzelhaft zugunsten des produktiveren Systems gemeinsamer Arbeit im Auburn-System wird von Hassemer, Steinert und Treiber nicht mit wirtschaftlichen, sondern mit ideologischen Gründen erklärt. Insofern bestreiten sie die Gültigkeit eines der beiden eindrucksvollsten Belege für die Arbeitsmarktthese.

Daß die Einzelhaft in Europa beibehalten wurde, scheint ihnen noch »etwas wirtschaftsnäher motiviert « (S. 39), obwohl ihnen der Gewinn an Disziplinierungspotential, den Einzelhaft bietet, das überzeugendere Argument zu sein scheint. Ihre Folgerung aus den verschiedenen historischen Anwendungssituationen der Theorie:

"Das Strafvollzugssystem hatte von der wirtschaftlichen Basis so weit abgehoben, daß an rein internen Problemen (z. B. disziplinären Schwierigkeiten) orientierte Lösungen offenbar durchsetzbar waren, auch wenn keinerlei wirtschaftliche Erwägungen für sie sprachen . . . Der Zusammenhang zwischen wirtschaftlicher Entwicklung und Strafrecht stellt sich damit in erster Linie auf dem Umweg über ideologische Entwicklungen her, die ihrerseits der wirtschaftlichen Entwicklung entsprechen« (S. 39).

Problematisch an diesem Fazit ist, daß stillschweigend davon ausgegangen wird, es gäbe ein einheitliches Erklärungsschema für Veränderungen im Strafrecht, im Strafprozeßrecht und im Strafvollzug. Aber Anwendungsvoraussetzungen, Zuweisungsregeln und Gestaltungsprinzipien für Strafe berühren die Interessen der gesellschaftlichen Klassen und Gruppen mehr oder weniger direkt. Durchsetzung bzw. Abwehr von Kriminalisierungen (Strafrechtsänderung) sind genuine Themen politischer Machtkämpfe; erst ein Unterliegen darin begründet Interessen an Prozeß- 
und Strafvollzugsgestaltung. Insoweit kommt ideologischen und politischen Erklärungen für Strafgestaltung ein geringerer Stellenwert zu als etwa für Strafrecht. Inhaltlich hat hierzu Melossi die entscheidenden Gedanken formuliert. Bezogen auf die drei disputierten Beispielsfälle Strafphilosophie der Aufklärung, Scheitern der Einzelhaft in den USA und Perpetuierung der Einzelhaft in Europa bietet er folgende ökonomischen Interpretationen an: Melossi weist auf Parallelen zwischen der Strafphilosophie des Beccaria, die das aufstrebende Bürgertum im Zeitalter der Aufklärung zu seiner eigenen machte, und einem wichtigen Aspekt der Entwicklung des bürgerlichen Subjekts hin. Nicht nur ist die Sicherheit des Eintretens von Strafe, auf der Beccaria vor allem als Abschreckungsvoraussetzung besteht, ein polemisches Argument gegen die Strafwillkür des Absolutismus (vgl. auch R \& K, S. III). Wichtiger: Beccarias Forderung nach einer Relation zwischen Tat und Strafe verkörperte, so formuliert Melossi im Anschluß an Paschukanis, eine Parallele zum bürgerlichen Zeitbegriff. Paschukanis schreibt:

"Die Entziehung der Freiheit auf eine im gerichtlichen Urteil vorher festgesetzte bestimmte Frist ist die spezifische Form, in der das moderne, d. h. bürgerlich-kapitalistische Strafrecht das Prinzip der äquivalenten Vergeltung verwirklicht. Diese Form ist unbewußt aber tiefliegend mit der Vorstellung vom abstrakten Menschen und von der abstrakten, durch Zeit meßbaren menschlichen Arbeit verbunden. Es ist kein Zufall, daß sich diese Form der Strafe gerade im 19. Jh. einbürgerte und als natürlich betrachtet wurde, d. h. zu einer Zeit, wo die Bourgeoisie alle ihre Eigenarten vollkommen entwickeln und erhärten konnte* (S. 165).

Für Melossi ergibt sich aus der Abstraktion, die das Prinzip der Schuldangemessenheit von Strafe darstellt, also eine ähnliche Verdinglichung menschlicher Existenz wie die Standardisierung von Lebenszeit als Arbeitszeit an Maschinen. Beides sind Mechanismen zur Herstellung einer dem Kapitalismus gemäßen Existenzauffassung.

Einen weiteren Mechanismus stellt die Kategorie der Disziplinierung dar. Melossi fragt, warum in der industriellen Revolution bei immensem Überfluß an Arbeitskraft entgegen der Arbeitsmarktthese an der Haft als wesentlicher Strafe festgehalten wurde, wobei ihre Gestaltung zur Tortur (Einzelhaft, Auspeitschen, Einführung der Tretmühle, kargste Ernährung) dem Verschlechterungsprinzip gemäß - also insofern im Sinne der Theorie - verkam. Seine These ist, daß die von Drill und von Bestrafung der geringsten Unkorrektheit geprägte Gestaltung der Haftstrafe im 18. und 19. Jh. eine kohärente Exekution der Disziplinierung war, wie sie in Fabriken realisiert wurde:

"Begriff und Praxis der Disziplin wurden zum Substrat kapitalistischer Arbeitsorganisation, wie sie sich in dieser Zeit entwickelte. Die Verbreitung der Disziplin aus der Fabrik heraus repräsentierte gleichzeitig die Verbreitung kapitalistischen Managements über die Gesamtheit bürgerlicher Sozialbeziehungen « (S. 75 ; eig. Übersetzung).

Einen ähnlichen Gedanken findet man auch bei Foucault: Gleichsetzung von Fabrik und Gefängnis (1977, S. 264). Auch eine Analyse des englischen Gefängniswesens während der Konstitutionsphase des Kapitalismus zeigt, daß die Gestaltung der Gefängnisdisziplin unter Einfluß maßgeblicher »Väter des Fabriksystems und Managements erfolgte (Ignatieff 1978, S. 62). Die in der Zeit der französischen Revolution entwickelten Strafphilosophien waren von simplem Materialismus geprägt. Bentham betrachtete das Panopticum als "Maschine, die den Schurken zum ehrlichen Menschen macht « (Ignatieff I 978, S.69). Weil Maschinen ständig verbessert und produktiver gemacht werden konnten, erwartete man analog vom Menschen, durch Drill seine Produktivität ebenso steigern zu können.

Diese Ideen hielten auch - über die Quäker in Philadelphia - Einzug in die Gestaltung des amerikanischen Strafvollzuges. Im Geiste der protestantischen Ethik propagier- 
ten sie erfolgreich die sittenbildende Einzelhaft, bei der die Gefangenen für eine geraume Zeit, ohne Personen zu sehen, ohne ein Buch zu lesen, ohne Werkzeuge zu haben, mit sich allein gelassen wurden. Wenn später dann die Arbeit auf der Zelle erlaubt wurde, "war sie keine unterdrückende Form der Strafe, sondern eine willkommene Ablenkung, Erbauung statt Last" (Rothman 1971 S. 86). Diesem pennsylvanischen System, Arbeit zur geliebten Routine werden zu lassen, stand das Auburn-System gegenüber, das Isolation bei Nacht in Einzelzellen vorsah, tagsüber aber strenges Schweige- und Kontaktverbot bei gemeinsamer Arbeit abverlangte und mit harten Strafen durchsetzte. Vehement wurde zwischen den Anhängern beider Systeme gestritten, welches von ihnen Vorbild für die vielen Gefängnisneubauten der USA sein sollte. Die gleiche Erfolglosigkeit beider Systeme erlaubre dann, dem Auburn-System den Vorzug zu geben: die Gefängnisse waren billiger zu bauen und erlaubten größere Einkünfte aus Gefangenenarbeit (Rothman 1971, S. 88). Ausschlaggebend mag aber auch gewesen sein, daß die Aufrechterhaltung des Schweigeverbots bei gemeinsamer Arbeit in immensem Umfang Strafen (Auspeitschungen, eiserne Knebel, Ankettung) notwendig machte, oder besser - zuließ. Zur Strafphilosophie gehörte die Disziplinierung, die Erzwingung totaler Unterordnung (Rothman 1971, S. 103 f.). Darüber hinaus arbeiteten die Gefangenen pro Woche 60 Stunden und erwirtschafteten immerhin gewisse Profite über die gesamten Betriebskosten hinaus (vgl. Miller 1974, S. 97 und vor allem S. 100). Soweit ersichtlich, gibt es also für die Situation in den USA des 19. Jh. hinreichende Belege dafür, daß Profiterwägungen die Gestaltung des Strafvollzuges beeinflußten. Andrerseits wird für die Epoche des Merkantilismus der ökonomische Nutzen von Gefangenenarbeit unterschiedlich beurteilt, und zwar schon - wie Stangl betont - bei v. Hippel (1 898, S. $468 \mathrm{f}$.), auf den sich $\mathrm{R} \& \mathrm{~K}$ zwar beziehen, aber ohne dessen $\mathrm{Zweifel}$ an der Rentabilität der Zuchthäuser zu beachten (Stangl, persönl. Mitteilung, 1980). Angesichts der Schwierigkeiten, empirische Belege für die Stichhaltigkeit eines Profitmotivs bei der Unterhaltung und Gestaltung von Gefängnissen für verschiedene Zeitpunkte und verschiedene Anstalten in genügendem Umfang aufzufinden, muß für den Augenblick die Frage der Profitorientierung des Strafvollzuges dahinstehen.

Sicherlich erschöpft sich im Profitaspekt aber nicht der Zusammenhang zwischen Produktionsverhältnissen und Strafgestaltung. Die Komplexität der Beziehungen zwischen Gefängnis und kapitalistischer Okonomie ergibt sich schon aus folgenden Hinweisen:

( $\mathrm{r})$ Da der Entwicklungsstand der Produktionsstätten in den Gefängnissen wegen des erhöhten Sabotage- und Zerstörungsrisikos, das Zwangsarbeit immer mit sich bringt, ein gewisses Niveau nicht überschreiten kann, sinkt die relative Produktivität der Gefängnisarbeit im Verlauf der Geschichte immer weiter ab im Vergleich zur Arbeit draußen; Gefängniszwangsarbeit wird immer weniger rentabel. (2) $\mathrm{Da}$ sich die Bewertung der Arbeitskraft von gefangenen und freien Arbeitern nach den Entwicklungen auf dem Arbeitsmarkt richtet, wächst bei Arbeitskräftemangel die Bereitschaft, Gefangene außerhalb der Gefängnisse zur Arbeit einzusetzen. Die Lebensverhältnisse im Gefängnis nehmen Arbeitskraftvernichtung nicht ohne weiteres in Kauf. Bei Überfluß an Arbeitskräften wird Reproduktion der Arbeitskraft der Gefangenen aber irrelevant, und in der Vollzugsgestaltung entsteht Raum für die ritualistische Demonstration von gesellschaftlichen Werten wie Disziplin, Untertanengeist, Arbeitsethik. (3) Darüber hinaus ist der Zusammenhang zwischen Arbeitsmarkt und Strafvollzug von ideologischen und politischen Einflußgrößen abhängig, die sich aus dem Stand der Klassenauseinandersetzung ergeben. Dieser Punkt wurde von Rusche schon in seinem frühen Aufsatz betont. Weil die Geschichte des 
Strafwesens eine „Geschichte der >zwei Nationen , wie sie Disraeli nannte (sei), aus

denen sich die Völker zusammensetzen, der Reichen und der Armen « (Rusche 1933, S. 70), gäbe es in diesem Kampf auch Situationen, wo weitere Verschärfungen der Strafen als "offene Provokation die ohnehin revolutionäre Situation zu überspannen " (Rusche 1933 , S. 74) drohten. Auch könne - wie in der Weimarer Republik - ein politisches Bündnis mit Schichten vorliegen, »die an dem Lose der Verbrecher interessiert waren « (Rusche 1933, S. 76) und »die Theorie von der gesellschaftlichen Verantwortlichkeit für das Verbrechen« (R \& K, S. 230) teilen.

3. Erklärungsschwächen für den Strafvollzug im 20. Jahrhundert

Diese drei Hinweise mögen darüberhinaus verdeutlichen, daß eine schlichte Fortschreibung der Arbeitsmarktthese für das 20. Jahrhundert nicht viel Erklärungskraft verspricht, wenn einmal unterstellt wird, daß Monopolbildung, weitgehend stabile Arbeitslosenrate und staatliche Sozialpolitik eine simple mechanistische Koppelung des Gebrauchs von Zwangsarbeit im Strafvollzug an den Arbeitsmarkt ausschließen. "Die Entstehung einer starken Gewerkschaftsbewegung forderte gerade den "Automatismus « des Arbeitsmarkts heraus « (Melossi 1978, S. 80), der die zyklische Minderbewertung der Arbeit voraussetzte.

Melossi wirft nun Kirchheimer vor, in den von ihm allein verfaßten Kapiteln $9^{-1} 3$ versäumt zu haben, eine Differenzierung des ökonomischen Erklärungsmodells, das für den frühen Kapitalismus ergiebig war, für die späteren Entwicklungsstadien zu liefern (Melossi 1978, S. 78). Statt dessen enthalten diese Kapitel staatstheoretische Analysen zur Rechtspolitik der Weimarer Republik. Und wo, wie in Kapitel Io, die ökonomischen Bedingungen der Bestrafungspolitik im 20. Jhd. behandelt werden, treten fiskalische Erklärungen an die Stelle der arbeitsmarktbezogenen. Kirchheimer hat offensichtlich eine Vorliebe für fiskalische Erklärungen des Umfangs und der Art der Anwendung von Freiheitsstrafe (vgl. R \& K, S. 2 I 2, S. 230, S. 242); »die Ende des r 8. Jahrhunderts entstandene Tendenz, den Strafvollzug als einen Verlustposten des Staatshaushalts zu betrachten « (R \& K, S. 244), wird durch verschiedene Reformen, vor allem die wachsende Verwendung von Geldstrafen, abzuschwächen versucht. Eine fiskalpolitische Erklärung der Verhältnisse im Strafvollzug ist allerdings ebenso formal und ausfüllungsbedürftig wie die Verschlechterungsthese: Da es zu Gefängnissen im Wohlfahrtsstaat eine Reihe von institutionellen und nicht-institutionellen Äquivalenten gibt (Arbeitslosengeld, Sozialhilfe, Obdachlosenasyle, Heime, psychiatrische Anstalten usw.), die alle als soziale Unkosten des Staates zu Buche schlagen (O'Connor 1974, S. 17, S. 182 ff.), wären Veränderungen in ihrer Zusammensetzung und Schwankungen innerhalb der Dimension, die die Finanzkrise des Staates jeweils hat, in der Erklärung zu berücksichtigen.

Die These Kirchheimers, der Geldstrafe würde im Spätkapitalismus eine wachsende Bedeutung zukommen, hat Jancovic für die USA widerlegt (1977, S. I 8). Die häufigste Strafe ist - wenigstens in den USA - Gefängnisstrafe mit oder ohne Bewährung, in dieser Reihenfolge. Folgerichtig fragt Jancovic, ganz im Sinne von R \& K, (a) inwieweit auf Bewährung ausgesetzte Freiheitsstrafen den Produktionsverhältnissen im Spätkapitalismus entsprechen und (b) worin, angesichts der Fortdauer der Gefängnisstrafe, deren Funktion für die kapitalistische Wirtschaft liegen könne. Seine Antworten sind: (a) Der Wandlung des spätkapitalistischen Arbeitsmarkts vom produktiven Sektor zum Dienstleistungssektor sei die Beziehung zwischen Bewährungshelfer und "Klient « völlig gemäß, denn die Betreuung sei eine Dienstleistung. Darüber hinaus erlaube sie, den Arbeitsplatz in der Gemeinde zu behalten. (Der letzte Punkt scheint mir allein der stichhaltige zu sein; wer wem in der Bewährungshilfsbeziehung zu Diensten ist, ist nämlich die Frage!). (b) Zur Funktion 
der Gefängnisstrafe heute weist Jancovic auf das Erfordernis hin, eine Reservearmee von Arbeitskräften zu unterhalten, deren Größe teilweise durch die Gefängnisse manipuliert werden könne (1977, S. 20).

Jancovic' Analyse wirft wiederum die Frage auf, inwieweit dem Strafsystem eine Funktion hinsichtlich der quantitativen Konstitution des Arbeitsmarkts zuerkannt werden kann. Auch hier scheint Melossi's Interpretation zutreffend zu sein, es müsse zunächst einmal um die Bestimmung qualitativer Zusammenhänge gehen. Gerade bezüglich der Bewährungsstrafe ist ja auf das in Umfang und Flexibilität, die Möglichkeiten des Gefängnisses (abgesehen vom Freigangvollzug) übersteigende, Disziplinierungspotential des Arbeitsverhaltens hinzuweisen, das nicht selten durch gute Kooperation zwischen Arbeitgeber und Bewährungshelfer gestützt wird. Und selbstverständlich entspricht der zunehmende Gebrauch von Bewährungsstrafen dem Erfordernis, wo die Voraussetzungen vorliegen, Facharbeiter nicht durch Einweisung in simple Gefängnistätigkeiten zu dequalifizieren. Es scheint, daß neue Strukturen des Arbeitsmarkts neue Formen der Strafen erfordern, wenn auch die spezifischen Bedingungen und Zusammenhänge erst noch aufgedeckt werden müssen

\section{Einige Hinweise zur Präzisierung des Ansatzes}

Die Sichtung der Diskussionsbeiträge zu R \& K's Analysen hat ergeben, daß der Zusammenhang zwischen Arbeitsmarkt und Strafvollzug keineswegs auf den Aspekt der Ausschöpfung oder quantitativen Manipulation des Arbeitskräftepotentials beschränkt werden darf, auch wenn $\mathrm{R} \& \mathrm{~K}$ so verstanden werden können. Ebenso verkürzt wäre es, die Gestaltung der Lebensverhältnisse durch Strafe nur unter Aspekten von Profitabilität oder Kostenminimierung zu sehen. Vielmehr muß die Organisation staatlichen Strafens nach Inhalt und Umfang als Teil der verschiedenen Mechanismen zur Herstellung des der kapitalistischen Produktionsweise gemäßen Menschen angesehen werden. Wie dies im einzelnen geschieht, bleibt einem Untersuchungsprogramm, das sich an R \& K orientiert, erst noch vorbehalten. Schon jetzt lassen sich aber, für die gegenwärtige Situation, einige Konkretisierungen machen.

(I) Was im Strafvollzug passiert, hat keine Demonstrationswirkung nach außen in dem Sinne, daß eine beispielhafte Disziplinierung stattfände, die Modellcharakter für die Disziplinierung draußen hätte. Was in den Gefängnissen passiert, bleibt der Offentlichkeit weitgehend verborgen. Solange nicht bezweifelt wird, daß Gefängnisaufenthalt eine Schlechterstellung der Lebensumstände bedeutet, brauchen Einzelheiten nicht mitgeteilt zu werden. Insofern geht jedenfalls heute vom Gefängnisleben kein gestalterischer Effekt auf die Lebensverhältnisse draußen in dem Sinne aus, daß sie sozusagen Bühnen wären, auf denen der ideale Arbeiter geformt und vorgeführt würde. Nicht auszuschließen ist, daß in einem Land mit sehr hohem Prisonierungsgrad etwa bei einer überproportional davon betroffenen Subpopulation, sofern sie intakte informelle Kommunikationsnetze besitzt, eine Vermittlung der Disziplinierung in den Gefängnissen auch nach draußen stattfindet. Aber im allgemeinen ist davon auszugehen, daß Inhaftierung primär sehr real bloß auf Unschädlichmachen von Personen auf Zeit zielt. Melossi's These, die Haftstrafe habe im 18. und 19. Jahrhundert überlebt, weil sie modellhaft die Disziplinierung des Fabrikarbeiters erlaubte, kann wohl nicht so verstanden werden, als käme dem Gefängnis eine substantielle Rolle als Sozialisationsagenten zu. Dazu müßte aus jeder Arbeiterfamilie zumindest ein Mitglied Hafterfahrung haben und weitergeben; solche Relationen sind aber im 20. Jh. keineswegs mehr gültig. In den Vollzugsverhältnissen mögen sich 
die auf die Organisation der Arbeitsverhältnisse bezogenen Werte wiederfinden, womöglich sogar in Perfektion und Reinkultur, aber nur als Reflex, nicht als Schaukasten.

(2) Damit hängt zusammen, daß es nicht sehr sinnvoll wäre, noch nach einer Funktion der Gefängnisse für die spätkapitalistische Gesellschaft in einem positiven Sinne zu suchen. Es gibt heute wohl keine Aufgabe innerhalb der Gesellschaft, die - gäbe es Gefängnisse nicht schon - zu ihrer Erfindung drängen würde. Damit ist nicht gemeint, Gefängnisse seien entbehrlich geworden. Es gibt immer noch genug Aufgaben für sie, die weitgehend der Ordnungsfunktion des Staates dienen: bestimmte politische und gesellschaftliche Störenfriede zeitweise aus dem Verkehr zu ziehen, das Machtgefälle zwischen den Rechten des Staates und den Rechten der Bürger zu demonstrieren, zur Abfuhr der kollektiven Strafbedürfnisse der sich selbst disziplinierenden Bevölkerung bereitzustehen, die Illusion, daß die größten Schädiger der Gesellschaft identifiziert und kontrolliert seien, zu perpetuieren usw. (vgl. Mathiesen 1979, S. I 57 f.). Diese Aufgaben könnten indessen wohl auch andere Kontrolltechniken (strikte Überwachung in Freiheit, öffentliche Bloßstellung in den Medien, Berufsverbote usw.) mittlerweile übernehmen.

(3) Setzt man die Austauschbarkeit der sozialen Kontrolltechnik Gefängnis durch andere voraus, kommen die verschiedenen, vor allem in den USA entwickelten Alternativen zum Strafvollzug in den Blick, deren maßgebliche Gemeinsamkeit darin zu bestehen scheint, daß die Kontrolle des Wohlverhaltens nach draußen verlegt wurde. Bewährungsstrafe, Führungsaufsicht, Meldeauflagen, Teilnahme an Kursen und Programmen, Weisungen zur Lebensführung - all das sind Formen, im täglichen Leben zusätzliche Kontrollrechte für Dritte zu begründen und damit Úberwachungsgürtel unterschiedlicher Dichte um bestimmte Personengruppen zu legen. Stanley Cohen hat diese Tendenz zur Herausverlagerung der Kontrolle aus dem Gefängnis als Trend zur "punitive city «, zur Strafanstalt Wohngemeinde, gegeißelt (1979, S. 357 f.). Dem Gefängnis bliebe dann nur noch die Aufgabe, Rückgrat einer sich immer weiter entfaltenden Palette von Kontrollen zu sein, das für den Notfall, wo nichts mehr sonst hilft, als Sicherheit zur Verfügung steht: als intervallartige Verschärfung der üblichen Kontrollformen.

(4) Der Beitrag des Gefängnisses zur Reproduktion des Arbeitsmarktes, der aber nicht schon seine Fortdauer rechtfertigt, kann nur darin gesehen werden, daß es die Rekrutierungsmechanismen für bestimmte Qualifikationsebenen abstützt, die Schule und Berufsausbildung jeweils zu leisten haben. Dabei spielt die Kriminalisierung jugendlicher Verfehlungen eine besondere Rolle. Ein großer Teil der Jugendkriminalität sind Bagatelltaten mit Einmaligkeitscharakter; die gnädige Reaktion des Jugendrichters, der sogenannte "Schuß vor den Bug« reicht hin, die Jugendlichen künftig zur Anpassung an die ihnen eröffneten niedrigen, mittleren und gehobenen Lebensmöglichkeiten und Karrierechancen zu bewegen. Ein anderer Teil der Jugendkriminalität kann als Protestverhalten jener Jugendlichen gewertet werden, denen in der Schule durch Sitzenbleiben und Abschiebung in Sonderschulen die Weichen in Richtung auf die niedrigsten Arbeitsqualifikationen gestellt werden. Jenen, denen durch die Kette jugendrichterlicher Weisungen, Arbeitsauflagen und schließlich Arreste nicht beigebracht werden kann, daß es besser wäre, die hochfliegenden Lebenserwartungen an das bereitgestellte tiefe Niveau der Lebenschancen anzupassen, stehen in den Jugendgefängnissen dann Nachhilfestunden in der Einübung von Hilfsarbeit bevor. Allerdings ergeben sich daraus nicht überwiegend brave Hilfsarbeiter, sondern Menschen, die ihr Leben teilweise in Gefängnissen verbringen müssen; dies sind dann gewissermaßen Kosten der Reproduktion der untersten Qualifikationsebene des Arbeitsmarkts. 
(5) Erwägenswert scheint mir schließlich, das Momentum der Vorstrafe an vorderster Stelle in die Analyse mit einzubeziehen. Daß Vorbestrafte bei Arbeits- und Wohnungssuche und auch sonst im sozialen Leben benachteiligt und diskriminiert werden, ist keineswegs nur auf Verständnislosigkeit und Ignoranz in der Bevölkerung zurückzuführen. Die Bevölkerung weiß sehr genau, worum es geht, und spielt den ihr zugedachten Part korrekt. Staatliche Behörden diskriminieren, stärker als Privatunternehmer, Vorbestrafte. Die Registrierung von Vorstrafen ist in Gesetzen verankert und durch Informationssysteme perfektioniert. Wäre die Diskriminierung Vorbestrafter unerwünscht, müßten die staatlichen Behörden als erste sich im Vergessen üben, Strafen löschen und Vorbestrafte einstellen. Sie müßten öffentlich aufklären, daß mit der Strafverbüßung reiner Tisch gemacht und alles vorbei sei. Das Gegenteil dieser Situation ist der Fall, und die Perfektionierung der Informationsverbreitung über Vorstrafen durch Auskunftspflicht des Vorbestraften und hinausgezögerte Fristen bis zur Löschung von Registereinträgen deuten an, daß weniger die Strafen als die Vorstrafen Signifikanz für den Arbeitsmarkt erlangen: zu klären wäre also der Zusammenhang zwischen ökonomischen Bedingungen und dem Diskriminierungsdruck, der zwischen Gefängnis und H.lfsarbeit pendelnde Existenzen erzeugt. A ber auch hier ist die quantitative Erheblichkeit dieses Zusammenhangs um so weniger einzuschätzen, je geringer es sich um abgrenzbare nationale Arbeitsmärkte handelt.

Die Zusammenhänge zwischen Arbeitsmarkt und Strafvollzug sind also nur auf dem Hintergrund sämtlicher Mechanismen zu verstehen, mit denen ein differenziertes Angebot an Arbeitskraft gesellschaftlich reproduziert wird. So verstanden bezeichnet der Ansatz von Rusche und Kirchheimer die Leitlinie eines noch ausstehenden Forschungsprogramms zum Zusammenhang zwischen Produktionsverhältnissen und Systemen sozialer Kontrolle.

Karl F. Schumann

\section{Literatur}

Stanley Cohen 1979: The Punitive City: Notes on the Dispersal of Social Control, in: Contemporary Crises, Vol. 3, S. 339-363.

Michel Foucault 1978: Uberwachen und Strafen, Frankfurt: stw 184.

W. Hassemer, H. Steinert, H. Treiber 1978: Soziale Reaktion auf Abweichung und Kriminalisierung durch den Gesetzgeber, in: W. Hassemer, K. Lüderssen (Hrsg.), Sozialwissenschaften im Studium des Rechts, München: Beck.

Rudolf v. Hippel 1 898: Beiträge zur Geschichte der Freiheitsstrafe, in: Zeitschrift für die gesamte Strafrechtswissenschaft, Bd. x8, S. 419-494, 608-662.

Michael Ignatieff 1978: A Just Measure of Pain. The Penitentiary in the Industrial Revolution, New York: Pentheon.

Ivan Jancovic 977: Labor Market and Imprisonment, in: Crime and Social Justice, No. 8, S. 17-31.

Thomas Mathiesen 1979: Uberwindet die Mauern! Neuwied: Luchterhand.

Dario Melossi 1978: Georg Rusche and Otto Kirchheimer: Punishment and Social Structure, in: Crime and Social Justice, No. 9, S. 73-85.

Martin B. Miller 1974: At Hard Labor: Rediscovering 19th Century Prison, in: Issues in Criminology, Vol. 9, S. 91-I r4.

Jessica Mitford 1971: Kind and Usual Punishment. New York: Vintage V-93.

James O'Connor 1974: Die Finanzkrise des Staates. Frankfurt: stw 83.

Leif Petter Olaussen 1976: Avspeilet straffen arbeidsmarkedssituasjonen? in: Sosiologi i dag, Vol. 4, S. 32-40. 
Eugen Paschukanis 1970: Allgemeine Rechtslehre und Marxismus (1929), Frankfurt:

Verlag Neue Kritik.

Karin Rausch 1975: Georg Rusche/Otto Kirchheimer, Sozialstruktur und Strafvollzug (Buchbesprechung), in: Kritische Justiz, S. 2 I 5-2 I 8.

David J. Rothman 197 I : The Discovery of the Asylum. Social Order and Disorder in the New Republic, Boston: Little, Brown \& Co.

Georg Rusche 1933: Arbeitsmarkt und Strafvollzug, in: Zeitschrift für Sozialforschung, Bd. 2, S. $63-78$.

Georg Rusche, Otto Kirchheimer 1974: Sozialstruktur und Strafvollzug (1939), Frankfurt: EVA.

Heinz Steinert, Hubert Treiber 1978: Versuch, die These von der strafrechtlichen Ausrottungspolitik im Spätmittelalter »auszurotten«. Eine Kritik an Rusche/ Kirchheimer und dem Okonomismus in der Theorie der Strafrechtsentwicklung, in: Kriminologisches Journal, 10. Jg., S. 81-106.

\section{Wissenschaftliche Legitimationen in der juristischen Ausbildungsreform durch Begleitforschung?}

Die Debatte um die Reform der Juristenausbildung 'ist, wie jedermann bekannt, ins Stocken geraten. Statt dessen hat eine andere Art von Reformpolitik Raum gewonnen, die die künftigen politischen Entscheidungsprozesse über die Juristenausbildung nach Ablauf der Experimentierphase vorzubereiten sucht. Diese neue Reformpolitik bedient sich des Mittels der »Evaluation«, der Sammlung empirisch fundierter Erfahrung zur traditionellen und zur reformierten Juristenausbildung. Mein Beitrag möchte deutlich machen, daß in die derzeit laufende Evaluation inhaltliche Vorgaben, forschungsorganisatorische Bedingungen und Vorentscheidungen gegenüber entwickelteren Ansätzen eingehen, die schon von der Gesamtanlage der zentralen Evaluation her nicht geeignet sind, Reformzielsetzungen zu fördern und die nötige Aufklärung über die Modelle und die bisherige Praxis der Juristenausbildung zu bringen. Auf der Ebene der Modell-Evaluation kommen weitgehend ungelöste Fragen von angemessener Begleitforschung hinzu.

I. Die Evaluationsvorhaben werden einerseits durch die Zentrale Forschungsgruppe zur Juristenausbildung in Mannheim, andererseits durch die sog. Gruppen vor Ort betrieben. Die Zentrale Forschungsgruppe wurde 1977 auf entsprechende Beschlïsse der Justizminister-Konferenz eingerichtet. Sie ist dem Unterausschuß zur Koordinierung der einstufigen Juristenausbildung des Reformausschusses der Justizminister-Konferenz unterstellt. Die Zentrale Forschungsgruppe soll die verschiedenen zur Erprobung gestellten Modelle untereinander und mit der herkömmlichen Ausbildung vergleichen und einen zusammenfassenden Bericht vorlegen. Sie soll

\footnotetext{
I Uber die hier nur gerafft darstellbaren Zusammenhänge informiert ausführlicher ein Beitrag von mir in den Materialien aus der Evaluation der Bremer Juristenausbildung, vv. Typoskript, Bremen April 1980, Nr. Is

2 Hesse, Hans-Albrecht, Uber den Stillstand der Debatte zur Reform der Juristenausbildung, JZ 1977 , 49-52 (49)
} 